\title{
ON SOME METHODS TO SOLVE INTEGRODIFFERENTIAL INVERSE PROBLEMS OF PARABOLIC TYPE
}

\author{
F. Colombo, Politecnico di Milano, Milan, Italy, fabrizio.colombo@polimi.it
}

In this paper we give an overview on some methods that are useful to solve a class of integrodifferential inverse problems. Precisely, we present some methods to solve integrodifferential inverse problems of parabolic type that are based on the theory of analytic semigroups, optimal regularity results and fixed point arguments. A large class of physical models can be treated with this procedure, for example phase-field models, combustion models and the strongly damped wave equation with memory to mention some of them.

Keywords: integrodifferential inverse problems; analytic semigroups.

This work is dedicated to the memory of Alfredo Lorenzi.

\section{Introduction}

Inverse problems arise in several fields of mathematics and physics. The heat equation with memory is among the most important models in the theory of inverse problems. In this equation the evolution of the temperature depends on the past thermal history of the material. To illustrate the problems associated with the heat equation with memory and the methods to solve them we recall the model.

Let $\Omega$ be an open bounded set in $\mathbb{R}^{3}$ and $T>0$. We can easily deduce the evolution equation for the temperature $u$ by the continuity equation

$$
D_{t} u(t, x)+\operatorname{div} J(t, x)-f(t, x, u(x, t), \nabla u(t, x))=0, \quad(t, x) \in[0, T] \times \Omega,
$$

in which the vector $J$ denotes the density of heat flow per unit surface area per unit time and $f$ is the heat source per unit volume per unit time in $\Omega$. The well known Fourier's law is given by

$$
J(t, x)=-D_{1} \nabla u(t, x)
$$

and its variation for materials with memory, supported by experiments, leads to replace (2) by

$$
J(t, x)=-D_{1} \nabla u(t, x)-D_{2} \int_{0}^{t} h(t-s, x) \nabla u(s, x) d s,
$$

where $D_{1}$ and $D_{2}$ are given positive functions and $h$ is the convolution kernel, which accounts for the thermal memory. To obtain the equation for the evolution of the temperature we replace (3) into the continuity equation (1) and we get

$$
D_{t} u(t, x)=\operatorname{div}\left[D_{1} \nabla u(t, x)+D_{2} \int_{0}^{t} h(t-s, x) \nabla u(s, x) d s\right]+f(t, x, u(x, t), \nabla u(t, x)) .
$$

The fundamental point, when we deal with memory effects is that the kernel $h$ cannot be considered a known function since there are no ways to measure $h$ directly. What we 
can do is to reconstruct $h$ by additional measurements on the temperature $u$ in a suitable subset of the body $\Omega$.

A possible additional information on $u$ can be represented in integral form as follows:

$$
\int_{\Omega} \phi(x) u(t, x) d x=g(t), \quad \forall t \in[0, T]
$$

where $\phi$ and $g$ are given functions representing the type of device used to measure the temperature and the outcomes of the measurements, respectively.

The inverse problem we consider in its more general form is the following.

Problem 1. Determine the temperature $u:[0, T] \times \Omega \longrightarrow \mathbb{R}$ and the convolution kernel $h:[0, T] \times \Omega \longrightarrow \mathbb{R}$ satisfying (4) and (5) under suitable initial-boundary conditions.

The general problem just introduced is very difficult to solve in the general form, but some approximated models of physical interest can be considered.

In the sequel we will consider the case of memory kernels that depend also on a space variable besides the time. The first results, regarding the identification of memory kernels depending on time and on a space variable, have been obtained with A. Lorenzi some years ago [1,2] and further developments can be found in [3-7]. There are several model that involve the heat equation with memory, for example phase-field models with memory, see [8-12] and the theory of combustion [13,14]. Other models can be found in [15], but the list is very long and there are many authors who have given important contributions. We have quoted just some of the papers where the strategy mentioned in the sequel applies. Finally, we recall that a model that can be treated with the methods of parabolic equations is the strongly damped wave equation with memory $[16,17]$.

To show the strategies to solve inverse problems we need to classify the nonlinearities that appear in the evolution equations. To better explain our methods we concentrate, at the moment, on the semilinear heat equation with memory kernel that depends on time, only:

$$
u_{t}(t, x)=\Delta u(t, x)+\int_{0}^{t} h(t-s) \Delta u(s, x) d s+f(t, x, u(t, x), \nabla u(t, x)),
$$

where $f$ is a given nonlinear function. We observe that the nonlinearities are of two types, since the kernel $h$ is unknown: the convolution term $\int_{0}^{t} h(t-s) \Delta u(s, x) d s$ contains the first type of nonlinearity, while the second term is obviously due to the nonlinear function $f(t, x, u(t, x), \nabla u(t, x))$.

Definition 1. (Classification of the nonlinearities) Let $u$ and $h$ be two unknown functions and let $A$ be an elliptic differential operator in the space variables (the Laplacian in most cases).

(a) We say that an inverse problem has nonlinearities of convolution type if it only contains nonlinearities of type $\int_{0}^{t} h(t-s) A u(s, x) d s$. 
(b) We say that an inverse problem has two types of nonlinearities if it contains nonlinearities of type $\int_{0}^{t} h(t-s) A u(s, x) d s$, and the term $F(u(t, x))$, where $F$ is a given nonlinear function of $u$ ( $F$ can depend also on some spatial derivatives of $u$ ).

Some inverse problems we will consider are associated with the evolution of the temperature in stratified materials with memory in which the stratification is along the direction of the $x$-axis:

$$
u_{t}(t, x, y)=\Delta u(t, x, y)+\int_{0}^{t} h(t-s, x) \Delta u(s, x, y) d s+f(t, x, y),
$$

for $(t, x, y) \in[0, T] \times[a, b] \times \omega \subset \mathbb{R}^{3}$ where $T>0, a<b$ and $\omega$ in a bounded set in $\mathbb{R}^{2}$. In this case, the kernel $h$ depends on the space variable $x$ and on time $t$. As we will see in the sequel, even if in this case the nonlinear term $f(u(t, x), \nabla u(t, x))$ is replaced by the known function $f(t, x, y)$ there are several difficulties that arise. Moreover the additional restriction on the temperature (5) must take into account the dependence on the axial variable $x$ as we will see.

We conclude this introduction recalling that there are several book on inverse problems, without claim completeness see [18-27].

\section{Some Methods to Solve Integrodifferential Inverse Problems}

The classification of the non linearities given in the introduction is valid for several physical models. According to such classification we will discuss the strategies to solve the inverse problems. First we recall the well known definition of well-posed problem in the sense of Hadamard which will be useful in the sequel.

Definition 2. Let $X, Y$ Banach spaces (often Sobolev spaces or spaces of continuous functions) and consider the equation

$$
T x=y,
$$

where $T$ is an operator from $X$ to $Y$. We say that $T x=y$ represents a well-posed problem in the sense of Hadamard if the following conditions hold:

- Uniqueness: $\forall y \in Y$ there is no more then one $x \in X$ satisfying (8).

- Existence: $\forall y \in Y$ there exists a solution $x \in X$ satisfying (8).

- Stability: Let $x_{j}(j=1,2)$ be solutions of (8) related to $y_{j}$, there exists $C>0$ such that

$$
\left\|x_{1}-x_{2}\right\|_{X} \leq C\left\|y_{1}-y_{2}\right\|_{Y}
$$

Let $\Omega$ be a bounded set in $\mathbb{R}^{3}$ and $T>0$. For the sake of simplicity we consider a simplified version of the evolution equation (for $t \in[0, T], x \in \Omega$ )

$$
u_{t}(t, x)=\Delta u(t, x)+\int_{0}^{t} h(t-s) \Delta u(s, x) d s+F(t, x), \quad t>0, \quad x \in \Omega \subset \mathbb{R}^{3},
$$


where $F$ is a given datum, the unknowns are $u$ and $h$ and we associate the following initial-boundary conditions:

$$
u(0)=u_{0}(x)
$$

and

$$
u(t, x)=0,(t, x) \in[0, T] \times \partial \Omega .
$$

We couple the evolution equation with the additional restriction on $u$ represented by (5). Since $h$ is unknown $\int_{0}^{t} h(t-s) \Delta u(s, x) d s$ is the only nonlinear term, so the inverse problem associated to (9) has a nonlinearity of convolution type only.

Remark 1. Several methods allows to consider additional measurements of flux type:

$$
\int_{S}\left(D_{\nu} u(t, x)+h * D_{\nu} u(t, x)\right) \sigma(d x)=g(t), \quad t \in(0, \tau),
$$

where $S$ is a subset of $\partial \Omega$, which is measurable with respect to the natural hypersurface measure $\sigma$ in $\partial \Omega$ and $\sigma(S)>0, g$ is a given function.

\subsection{Strategy to Prove Local in Time Results for the Inverse Problem with a Nonlinearity of Convolution Type}

The procedure is as follows.

(1) We consider an abstract formulation of the inverse problem relating it to a Banach space $X$.

(2) We choose a functional setting: for example we can take the Sobolev spaces on $[0, T]$ with values in the Banach space $X$ and we select the related optimal regularity theorem for the linearized version of the problem.

(3) We prove that the abstract version of the problem is equivalent to a suitable fixed point system.

(4) Since the fixed point system (second kind Volterra equations) contains integral operators, we have to estimate them in the spaces we are considering.

(5) By the Contraction Principle we prove that the equivalent problem has a unique solution, so we get existence and uniqueness of a solution to our inverse problem.

(6) We apply the abstract results to the concrete problem.

\subsection{Strategy to Prove Global in Time Results for Nonlinearity of Convolution Type}

The procedure is similar to the previous one buy with the use of weighted space we get global in time results. It is clear that the use of weighted space makes all the computations more involved. The procedure is as follows.

(1) We consider an abstract formulation of the inverse problem relating it to a Banach space $X$, to have a more general problem. 
(2) We choose a functional setting. For example, we can take the space of bounded functions on $[0, T]$ or the Sobolev spaces on $[0, T]$ with values in the Banach space $X$ and we select the related optimal regularity theorem for the linearized version of the problem.

(3) We prove that the abstract version of the problem is equivalent to a suitable fixed point system.

(4) Since the fixed point system contains integral operators, we have to estimate them in the weighted spaces we are considering (exponential weight $e^{\sigma t}, \sigma \in \mathbb{R}^{-}$or $e^{-\sigma t}$, $\sigma \in \mathbb{R}^{+}, t \in[0, T]$ is usually used).

(5) By the Contraction Principle we prove that the equivalent problem has a unique solution global in time.

(6) We apply the abstract results to the concrete problem.

\subsection{Local in Time Results for the Two Types of Non Linearities}

The same procedure, in Subsection 1.1 allows us to treat also problems in which there are the two types of nonlinearities. It is the case when $F$ depends on $u$. With this technique we obtain local in time results, see for example the papers $[1,2,8,12,13,15,28]$.

Remark 2. One of the main difficulties to get global in time results for inverse problems with both types of nonlinearities using strategy in Subsection 1.2 is that the weighted spaces work well for the non linearity of convolution type but it creates problems with the other types of non linearity. To avoid such problem we have found a strategy that does not make use of the weighted spaces.

\subsection{The Strategy to Find Global in Time Results for Inverse Problems with Two Types Nonlinearities with Growth Conditions}

This strategy has been developed in [29]. To explain the technique let us consider the simple model

$$
u_{t}(t, x)=\Delta u(t, x)+\int_{0}^{t} h(t-s) \Delta u(s, x) d s+F(u(t, x)) \text {, }
$$

where $F$ is a given nonlinear function and we consider the additional restriction on $u$ given by (5). So

$$
F(u(t, x)) \text { and } \int_{0}^{t} h(t-s) \Delta u(s, x) d s
$$

are the two nonlinearities one has to consider. The main ideas to solve the problem in this case is to prove that there exists a local in time solution of the inverse problem in Sobolev spaces without weights, then we "linearize the convolution term" and we find a priori estimates for $u$ and for the convolution kernel $h$. More precisely we proceed as follows. The method is related with the Sobolev spaces $W^{2, p}\left(0, T ; L^{p}(\Omega)\right)$. 
(1) Find a suitable equivalent fixed point system that contains integral operators. We have to estimate them in the Sobolev spaces we have chosen.

(2) We apply the Contraction Principle, to the fixed point system, to prove that there exists a unique local in time solution. Thanks to the equivalence theorem previously obtained we get existence and uniqueness of the solution to our inverse problem which is local in time.

(3) We prove a global in time uniqueness result without the condition that $F_{u}$ is globally bounded.

(4) We linearize the convolution term. This is possible thanks to the local in time existence and uniqueness theorem. In fact we observe that a unique solution $(\hat{u}, \hat{h})$ exists in $[0, \tau]$ for some $\tau>0$.

(5) We set $v(t):=u^{\prime}(t)$ and $v_{\tau}(t)=v(\tau+t)$ and $h_{\tau}(t)=h(\tau+t)$ and consider, for $0<t<\tau$ the splitting

$$
\int_{0}^{\tau+t} h(\tau+t-s) \Delta v(s, x) d s=h_{\tau} * \Delta \hat{v}(t, x)+\hat{h} * \Delta v_{\tau}(t, x)+\tilde{F}(t, x),
$$

where the symbol $*$ stands for the convolution. $\tilde{F}(t, x)$ is a given data and depends on the known functions $(\hat{v}, \hat{h})$. This way of rewriting the convolution term allows us to avoid the weighted spaces that have a bad behavior when we deal with the nonlinearity $F(u)$.

(6) We deduce a priori estimates for $v_{\tau}(t):=u_{\tau}^{\prime}(t)$ and $h_{\tau}(t)$ for $0<t<\tau$ with the condition $F_{u}$ globally bounded. In a finite number of steps we extend the solution to the interval $[0, T]$.

\section{Inverse Problems for Stratified Materials}

We now introduce some approximated physical models without giving the precise functional setting, since we are interested in pointing out the physical differences among the models. We will be more precise in the sequel when we introduce the functional setting.

Promblem 2. Given the data $\phi, g, u_{0}$ and $f$ determine the couple $(u, h)$ satisfying the system

$$
\begin{gathered}
u_{t}(t, x, y)=B u(t, x, y)+\int_{0}^{t} h(t-s, x) B u(s, x, y) d s+f(t, x, y), \quad(t, x, y) \in[0, T] \times \Omega, \\
u(0, x, y)=u_{0}(x, y), \quad(x, y) \in \Omega, \\
u(t, x, y)=0, \quad(t, x, y) \in[0, T] \times \partial \Omega, \\
\int_{\omega} \phi(y) u(t, x, y) d y=g(t, x), \quad(t, x) \in[0, T] \times[0, L]
\end{gathered}
$$

in the framework of Sobolev fractional order spaces.

Here $\Omega=[0, L] \times \omega$ is a bounded cylinder in the space variables $x \in[0, L]$ and $y \in \omega \subset \mathbb{R}^{2}, B$ is a uniformly elliptic differential linear operator, in particular $B$ is 
split in the sum $B_{1}+B_{2}$ and $B_{1}$ and $B_{2}$ commute in the sense of resolvent. In this first approximation we suppose that the term $\int_{0}^{t} \nabla h(t-s, x) \nabla u(s, x) d s$ can be neglected. The sum of applications has been introduced to formulate the problem in an equivalent fixed point form. The problem has been studied using the theory of analytic semigroups in the papers $[1,2]$.

Problem 3. Given the data $\phi_{1}, \phi_{2}, g_{1}, g_{2}, \ell, u_{0}$ and $f$ determine the couple $(u, h)$ satisfying the system

$$
\begin{aligned}
& \begin{array}{l}
u_{t}(t, x, y)=A u(t, x, y)+\int_{0}^{t} h(t-s, x) B u(s, x, y) d s+\int_{0}^{t} D_{x} h(t-s, x) C u(s, x, y) d s \\
\quad+f(t, x, y), \quad(t,(x, y)) \in[0, T] \times \Omega,
\end{array} \\
& \begin{array}{l}
u(0, x, y)=u_{0}(x, y), \quad(x, y) \in \Omega, \\
\partial_{\nu_{A}} u(t, x, y)=\ell(t, x, y), \quad(t,(x, y)) \in[0, T] \times \partial \Omega, \\
\int_{\Omega} \phi_{1}(x, y) u(t, x, y) d x d y=g_{1}(t), \quad t \in[0, T] \\
\int_{\omega} \phi_{2}(x, y) u(t, x) d y=g_{2}(t, x), \quad(t, x) \in[0, T] \times[0, L]
\end{array}
\end{aligned}
$$

in the framework of the space of continuous functions.

Here $\Omega$ is as above, $A$ and $B$ are second order operators, while $C$ is a first order linear differential operator. The novelty with respect to the first problem is that $A$ is split in the sum $A_{1}+A_{2}$ with $A_{1}$ and $A_{2}$ that do not commute in the sense of resolvent. This problem is a generalization of the one above also for the introduction of the term $\int_{0}^{t} D_{x} h(t-s, x) C u(s, x) d s$. The above inverse problem was studied in [3].

In the following we give the mathematical tools to study Problem 2 to give the flavor of the difficulties that there are behind. The second problem is more complicated.

We define the Sobolev spaces that will be used in the sequel to formulate our results. The Sobolev spaces of fractional order $W^{\sigma, p}((0, T) ; X)$ consist of all functions $f \in L^{p}((0, T) ; X)$ for which

$$
|f|_{W^{\sigma, p}((0, T) ; X)}:=\left(\int_{0}^{T} d t_{1} \int_{0}^{T}\left|t_{2}-t_{1}\right|^{-1-\sigma p}\left\|f\left(t_{2}\right)-f\left(t_{1}\right)\right\|^{p} d t_{2}\right)^{1 / p}<+\infty,
$$

$W^{\sigma, p}((0, t) ; X)$ turn out to be a Banach spaces when equipped with the norms

$$
\begin{gathered}
\|f\|_{W^{\sigma, p}((0, T) ; X)}=\left(\left\|t^{-\sigma} f\right\|_{L^{p}((0, T) ; X)}^{p}+|f|_{W^{\sigma, p}((0, T) ; X)}^{p}\right)^{1 / p}, \quad \text { if } \sigma \in(0,1 / p), \\
\|f\|_{W^{\sigma, p}((0, T) ; X)}=\left(\|f\|_{L^{p}((0, T) ; X)}^{p}+|f|_{W^{\sigma, p}((0, T) ; X)}^{p}\right)^{1 / p}, \quad \text { if } \sigma \in(1 / p, 1) .
\end{gathered}
$$

We now recall some results from the analytic semigroup theory and interpolation spaces. For more details see [30]. Let $B: D(B) \subset X \rightarrow X$ be a linear closed operator (possibly with $\overline{D(B)} \neq X$ ) satisfying the following assumptions: 
i) there exists $\theta \in(\pi / 2, \pi)$ such that the resolvent set of $B$ contains 0 and the open sector $\Sigma_{\theta}=\{\mu \in \mathbb{C} \backslash\{0\}:|\arg \mu|<\theta\} ;$

ii) there exists $M>0$ such that $\left\|(\lambda I-B)^{-1}\right\|_{\mathcal{L}(X)} \leq M|\lambda|^{-1}$ for any $\lambda \in \Sigma_{\theta}$.

Here $\mathcal{L}(X)$ denotes the Banach space of all bounded linear operators from $X$ into itself equipped with the sup-norm.

By virtue of assumptions i), ii) we can define the analytic semigroup $\left\{\mathrm{e}^{t B}\right\}_{t \geq 0}$ of bounded linear operators in $\mathcal{L}(X)$ generated by $B$.

After endowing $D(B)$ with the graph-norm we can define the following family of interpolation spaces $D_{B}(\beta,+\infty)$ (for $\beta \in(0,1)$ ), which are intermediate between $D(B)$ and $X$ :

$$
D_{B}(\beta,+\infty)=\left\{x \in X:|x|_{D_{B}(\beta,+\infty)}:=\sup _{t>0} t^{(1-\beta)}\left\|B e^{t B} x\right\|<\infty\right\} .
$$

Moreover, we set

$$
D_{B}(1+\beta,+\infty)=\left\{x \in D(B): B x \in D_{B}(\beta,+\infty)\right\} .
$$

Consequently, $D_{B}(n+\beta,+\infty)(n \in \mathbb{N}, \beta \in(0,1))$ turns out to be a Banach spaces when equipped with the norm

$$
\|x\|_{D_{B}(n+\beta,+\infty)}=\sum_{j=0}^{n}\left\|B^{j} x\right\|+\left|B^{n} x\right|_{D_{B}(\beta,+\infty)} .
$$

With any linear operator $B$ satisfying assumptions of type i), ii) we can associate the vector spaces $D_{B}(\eta, p)(\eta \in(0,1), p \in(1,+\infty))$, intermediate between $X$ and $D(B)$, defined by

$$
D_{B}(\eta, p)=\left\{x \in X:|x|_{\eta, p}:=\left(\int_{0}^{+\infty} t^{(1-\eta) p-1}\left\|(B-\omega I) \mathrm{e}^{t(B-\omega I)} x\right\|^{p} d t\right)^{1 / p}<+\infty\right\} .
$$

Analogously, we set for any $p \in(1,+\infty)$ and $\eta \in(0,1)$

$$
D_{B}(1, p)=D(B), \quad D_{B}(1+\eta, p)=\left\{x \in D(B): B x \in D_{B}(\eta, p)\right\} .
$$

We recall that $D_{B}(n+\eta, p)(n=0,1)$ are Banach spaces when equipped with the norms

$$
\|x\|_{n+\eta, p}=\sum_{j=0}^{n}\left\|B^{j} x\right\|+\left|B^{n} x\right|_{\eta, p}, \quad \eta \in(0,1), p \in(1,+\infty), n=0,1 .
$$

For any fixed pair of Banach spaces $X_{1}$ and $X_{2}$ we denote by $\mathcal{L}\left(X_{1} ; X_{2}\right)$ the space of all bounded linear operators endowed with the uniform norm. We also set $\mathcal{L}\left(X_{1} ; X_{1}\right)=\mathcal{L}\left(X_{1}\right)$.

Problem 2 can be reformulated in an abstract setting related to a Banach space $X$ as follows: find a function $u:[0, T] \rightarrow X$ and an operator $H:[0, T] \rightarrow \mathcal{L}(X)$ satisfying the equations:

$$
u^{\prime}(t)=\left(B_{1}+B_{2}\right) u(t)+\int_{0}^{t} H(t-s)\left(B_{1}+B_{2}\right) u(s) d s+f(t), \quad t \in[0, T],
$$




$$
\begin{gathered}
u(0)=u_{0}, \\
\Phi(u(t))=G(t), \quad t \in[0, T],
\end{gathered}
$$

where $\Phi$ is a bounded linear functional and $G$ and $u_{0}$ are given data. The main assumptions are the following. We now endow with the graph norms the domains $D\left(B_{j}\right) \subset X$ of the linear closed operators $B_{j}(j=1,2)$.

Further, we assume that $B_{j}: D\left(B_{j}\right) \subset X(j=1,2)$ are sectorial operators with resolvent sets $\rho\left(B_{j}\right)$, respectively, related to six constants $\omega_{j} \in \mathbb{R}, \theta_{j} \in(\pi / 2, \pi), M_{j}>0$ $(j=1,2)$ such that

$$
\rho\left(B_{j}\right) \supset S_{\theta_{j}, \omega_{j}}=\left\{\lambda_{j} \in \mathbf{C} ; \lambda_{j} \neq \omega_{j},\left|\arg \left(\lambda_{j}-\omega_{j}\right)\right|<\theta_{j}\right\}
$$

Moreover, we assume that:

(H3) there esists a pair $\left(\mu_{1}, \mu_{2}\right) \in \rho\left(B_{1}\right) \times \rho\left(B_{2}\right)$ such that

$D\left(\left(\mu_{1} I-B_{1}\right)\left(\mu_{2} I-B_{2}\right)\right)=D\left(\left(\mu_{2} I-B_{2}\right)\left(\mu_{1} I-B_{1}\right)\right)$

$\left(\mu_{1} I-B_{1}\right)\left(\mu_{2} I-B_{2}\right)=\left(\mu_{2} I-B_{2}\right)\left(\mu_{1} I-B_{1}\right)$;

(H6) $\rho\left(B_{1}+B_{2}\right)$ contains a real number $\mu>\max \left(0, \omega_{1}\right)+\omega_{2}$;

(H9) $H \Phi[u]=\Phi[H u], \quad \forall u \in X, \forall H \in \mathcal{K}$;

(H10) $H \in \mathcal{L}\left(D\left(B_{1}\right)\right)$ and $B_{2} H=H B_{2}, \quad \forall H \in \mathcal{K}$;

(H11) $\Phi\left(B_{1} u_{0}+B_{2} u_{0}-\mu u_{0}\right)$ is invertible in $\mathcal{K}$ for some $\mu \in\left(\max \left(0, \omega_{1}\right)+\right.$ $\left.\omega_{2},+\infty\right)$ and its inverse operator $\Lambda\left(u_{0}\right):=\left[\Phi\left(B_{1} u_{0}+B_{2} u_{0}-\mu u_{0}\right)\right]^{-1}$ belongs to $\mathcal{K}$.

We recall that $\mathcal{K}$ denotes a closed subalgebra in $\mathcal{L}(X) \cap \mathcal{L}\left(D\left(B_{1}\right) \cap D\left(B_{2}\right)\right)$.

We list our assumptions on the data

(K1) $\quad f \in W^{\sigma, p}\left(\left(0, T_{0}\right) ; D\left(B_{1}\right) \cap D\left(B_{2}\right)\right) \cap W^{1+\sigma, p}\left(\left(0, T_{0}\right) ; D\left(B_{2}\right)\right)$;

$(\mathrm{K} 2) \quad u_{0} \in D\left(B_{1}\right) \cap D\left(B_{2}\right) \cap D\left(\left(B_{1}+B_{2}\right) B_{2}\right), \quad u_{0}$ satisfies $H 11$

$$
G \in W^{2+\sigma, p}\left(\left(0, T_{0}\right) ; \mathcal{L}(X)\right) \cap W^{1+\sigma, p}\left(\left(0, T_{0}\right) ; D\left(B_{1}\right)\right)
$$


$(\mathrm{K} 4)$

$w_{0} \in D_{B_{1}}(\sigma+1 / p, p) \cap D_{B_{2}}(\sigma+1 / p, p) ;$

(K5) $\quad w_{1} \in D_{B_{1}}(\sigma-1 / p, p) \cap D_{B_{2}}(\sigma-1 / p, p)$, if $\sigma \in(1 / p, 1)$; where $T_{0}$ is a fixed positive number and

$$
\begin{gathered}
w_{0}:=\left(B_{2}-\mu I\right)\left[\left(B_{1}+B_{2}-\mu I\right) u_{0}+f(0)\right] \\
w_{1}:=\left(B_{1}+B_{2}-\mu I\right)\left(B_{2}-\mu I\right)\left[\left(B_{1}+B_{2}-\mu I\right) u_{0}+f(0)\right]+\left(B_{2}-\mu I\right) f^{\prime \prime}(0) \\
-\left\{\Phi\left[\left(B_{2}-\mu I\right)\left(\left(B_{1}+B_{2}-\mu I\right) u_{0}+f(0)\right)\right]-G^{\prime \prime}(0)+B_{1} G^{\prime \prime}(0)+\Phi\left[f^{\prime \prime}(0)\right]\right\} \Lambda\left(u_{0}\right) \\
\times\left(B_{2}-\mu I\right)\left(B_{1}+B_{2}-\mu I\right) u_{0} .
\end{gathered}
$$

Then we list the consistency conditions related to our problem

$$
\Phi\left[u_{0}\right]=G(0)
$$

$$
\left(B_{1}+B_{2}-\mu I\right) G(0)+\Phi\left[B_{2} u_{0}+f(0)\right]=G^{\prime}(0) .
$$

We denote by $\mathcal{G}$ the space of our admissible data satisfying (H1)-(H11) and (K1)-(K7). We also defined the following subset $\mathcal{G}(m)\left(m \in \mathbb{R}_{+}\right)$by

$$
\mathcal{G}(m)=\left\{\left(f, G, u_{0}\right) \in \mathcal{G}:\left\|\left[\Phi\left(B_{1} u_{0}+B_{2} u_{0}-\mu u_{0}\right)\right]^{-1}\right\|_{\mathcal{K}} \leq m\right\}
$$

and w define then the following Banach spaces, where $s \in[1,+\infty)$ :

$$
\mathcal{U}^{s, p}(X)=W^{s, p}((0, T) ; X) \cap W^{s-1, p}\left((0, T) ; D\left(( B _ { 1 } ) \cap D \left(\left(B_{2}\right) .\right.\right.\right.
$$

Finally, we give the main theorems:

Theorem 1. Let $\left(f, G, u_{0}\right) \in \mathcal{G}(m)$ for some $T_{0}>0, p \in(1,+\infty), m>0$, and let $\sigma \in(0,1) \backslash\{1 / p\}$. Then there exists $T^{*} \in\left(0, T_{0}\right]$ such that for any $T \in\left(0, T^{*}\right]$ problem (23)-(25) admits a unique solution $(u, H) \in \mathcal{U}^{2+\sigma, p}(X) \times W^{\sigma, p}((0, T) ; \mathcal{K})$.

Theorem 2. For any $m>0$ and $T \in\left(0, T^{*}\right]$ the map $\left(f, G, u_{0}\right) \rightarrow(u, H)$, where $(u, H)$ is the unique solution to problem (23)-(25), is bounded (i.e. maps bounded sets into bounded sets) and is Lipschitz continuous from $\mathcal{G}(m)$ into

$$
\mathcal{U}^{2+\sigma, p}(X) \times W^{\sigma, p}((0, T) ; \mathcal{K})
$$

The abstract theorems stated above can be applied to the concrete Problem 2 choosing as reference spaces $X=L^{p}\left(\Omega_{2} ; W_{0}^{1, p}\left(\Omega_{1}\right)\right)$ where $W_{0}^{1, p}$ are the well known Sobolev spaces and $p>1$. The chosen spaces seem to be unusual, in fact one could think that the most natural spaces are $X=L^{p}\left(\Omega_{1} \times \Omega_{2}\right)$, but this turns out to be not correct because the evolution equation does not have meaning here. The above choice is necessary, because the kernel $h$ depends on the spatial variable $x$. 


\section{Abstract Formulation of Inverse Problems with Two Types of Non Linearities}

We list what we need to state our results.

- $X^{\prime}$ stands for $\mathcal{L}(X, \mathbb{R})$.

- If $X$ and $Y$ are Banach spaces, with norms $\|\cdot\|_{X}$ and $\|\cdot\|_{Y}$ respectively, and $(x, y) \in$ $X \times Y$, we set

$$
\|(x, y)\|_{X \times Y}:=\|x\|_{X}+\|y\|_{Y} .
$$

- If $Y$ and $X$ are Banach spaces, $f: Y \rightarrow X$, and $y \in Y$, we shall indicate with $f^{\prime}(y)$ the Fréchet-derivative of $f$ in $X$.

- If $s \in \mathbb{Z}, s \geq 2$ and $\Omega$ is an open subset of $\mathbb{R}^{n}$, with smooth boundary, we set $W_{B}^{s, p}(\Omega):=\left\{f \in W^{s, p}(\Omega): D_{\nu} f \equiv 0\right\}$, with $D_{\nu}$ outward unit normal derivative in $\partial \Omega$.

- We denote by $B_{p, q}^{s}(\Omega)(s>0,1 \leq p, q \leq+\infty)$ Besov spaces (see $\left.[31,32]\right)$.

- The symbol $(\cdot, \cdot)_{\theta, p}$ stands for the real interpolation functor $(0<\theta<1,1 \leq p \leq$ $+\infty)$.

- Let $h \in L^{1}(0, T)$ and $f:(0, T) \rightarrow X$, where $X$ is a Banach space. We define the convolution

$$
(h * f)(t):=\int_{0}^{t} h(t-s) f(s) d s,
$$

whenever the integral has a meaning.

- In the sequel we will denote by $a \wedge b$ the number $\min \{a, b\}$ where $a, b \in \mathbb{R}$.

- Let $p \in[1,+\infty), T \in \mathbb{R}^{+}$, and let $X$ be a Banach space.

If $f \in W^{m, p}(0, T ; X)(m \in \mathbb{N}, p \in[1,+\infty])$ (see [33]), we set

$$
\|f\|_{W^{m, p}(0, T ; X)}:=\sum_{j=0}^{m-1}\left\|f^{(j)}(0)\right\|+\left\|f^{(m)}\right\|_{L^{p}(0, T ; X)} .
$$

- Let $X$ be a Banach space and let $A$ be a linear closed operator in $X$, with domain $D(A)$. We set, for $x \in D(A)$,

$$
\|x\|_{D(A)}:=\|x\|_{X}+\|A x\|_{X}, \quad x \in D(A) .
$$

If $u \in W^{k+1, p}(0, T ; X) \cap W^{k, p}(0, T ; D(A))$, we set

$$
\|u\|_{W^{k+1, p}(0, T ; X) \cap W^{k, p}(0, T ; D(A)),}=\|u\|_{W^{k+1, p}(0, T ; X)}+\|u\|_{W^{k, p}(0, T ; D(A))},
$$

where $W^{0, p}(0, T ; D(A)):=L^{p}(0, T ; D(A))$.

We solve the inverse problem under the following conditions on the data. Let $p \in(1,+\infty)$ and let $X, Y, D(A)$ be Banach spaces such that: 
(H1) $D(A) \hookrightarrow Y \hookrightarrow X, D(A)$ is dense in $X$ and there exist $C>0$ and $\theta \in[0,1)$, such that, $\forall u \in D(A)$,

$$
\|u\|_{Y} \leq C\|u\|_{X}^{1-\theta}\|u\|_{D(A)}^{\theta} .
$$

In general, if $x \in X$, we shall write $\|x\|$ instead of $\|x\|_{X}$.

(H2) $\forall T \in \mathbb{R}^{+}$, the Cauchy problem

$$
\left\{\begin{array}{l}
u^{\prime}(t)=A u(t)+f(t), \quad t \in(0, T) \\
u(0)=0
\end{array}\right.
$$

is well-posed in $L^{p}(0, T ; X)$, in the following sense: $\forall f \in L^{p}(0, T ; X),(31)$ has a unique solution $u$ belonging to $W^{1, p}(0, T ; X) \cap L^{p}(0, T ; D(A))$.

Remark 3. Assumptions $(H 1)-(H 2)$ imply that $A$ is the infinitesimal generator of an analytic semigroup in $X$ (see [34, Corollary 4.2]).

\subsection{The Strongly Damped Wave Equation}

Definition 3. Determine $\tau \in(0, T]$ and

$$
u \in W^{3, p}(0, \tau ; X) \cap W^{2, p}(0, \tau ; D(A)), \quad h \in L^{p}(0, \tau), \quad t \in(0, \tau)
$$

satisfying the system

$$
\begin{cases}u^{\prime \prime}(t)=A u^{\prime}(t)+A u(t)+h * B u(t)+f(u(t))+G(t), & t \in(0, \tau) \\ u(0)=u_{0}, & \\ u^{\prime}(0)=u_{1}, & t \in(0, \tau) . \\ \Phi(u)=g(t), & \end{cases}
$$

We solve the inverse problem under the following conditions on the data. Let $p \in(1,+\infty)$ and let $X, Y, D(A)$ Banach spaces such that (H1) and (H2) hold and assume that:

(H3) $B \in \mathcal{L}(D(A), X)$.

(H4) $u_{0}, u_{1} \in D(A)$.

(H5) $\Phi \in X^{\prime}$ and $D\left(A^{\prime}\right)$ is dense in $X^{\prime}$.

(H6) $f \in C^{1}(Y, X)$ and $f^{\prime}: Y \rightarrow \mathcal{L}(Y, X)$ is Lipschitz continuous on the bounded subsets of $Y$.

(H7) $G \in W^{1, p}(0, T ; X)$.

(H8) $v_{1}:=A u_{1}+A u_{0}+f\left(u_{0}\right)+G(0) \in(X, D(A))_{1-1 / p, p}$.

(H9) $\Phi\left(B u_{0}\right) \neq 0$.

(H10) $g \in W^{3, p}(0, T)$ with $\Phi\left(u_{0}\right)=g(0), \Phi\left(u_{1}\right)=g^{\prime}(0)$ and $\Phi\left(v_{1}\right)=g^{\prime \prime}(0)$.

(H11) $f^{\prime}: Y \rightarrow \mathcal{L}(Y, X)$ is bounded, with $f^{\prime}$ Fréchet derivative of $f$. 
Theorem 3. (Local in time existence). Let the assumptions (H1)-(H10) hold. Then there exists $\tau \in(0, T]$, depending on the data, such that the inverse problem given by Definition 3 has a solution $(u, h) \in\left[W^{3, p}(0, \tau ; X) \cap W^{2, p}(0, \tau ; D(A))\right] \times L^{p}(0, \tau)$.

Theorem 4. (Global in time uniqueness). Let the assumptions (H1)-(H10) hold. Let $\tau \in(0, T]$, and suppose that the inverse problem given by Definition 3 has two solutions $\left(u_{j}, h_{j}\right) \in\left[W^{3, p}(0, \tau ; X) \cap W^{2, p}(0, \tau ; D(A))\right] \times L^{p}(0, \tau) \quad(j \in\{1,2\})$, then $u_{1}=u_{2}$ and $h_{1}=h_{2}$.

Theorem 5. (Global in time existence and uniqueness). Let the assumptions (H1)-(H10) hold. Let $T>0$. Then the inverse problem given by Definition 3 has a unique solution $(u, h) \in\left[W^{3, p}(0, T ; X) \cap W^{2, p}(0, T ; D(A))\right] \times L^{p}(0, T)$.

Remark 4. We point out that the global in time existence and uniqueness result has been obtained in the abstract setting and it is not based on a maximum principle that in most of the concrete cases does not hold.

\subsection{Abstract Inverse Problem for the Parabolic Case}

Definition 4. Let $T>0$. Determine $\tau \in(0, T]$ and

$$
u \in W^{2, p}(0, \tau ; X) \cap W^{1, p}(0, \tau ; D(A)), \quad h \in L^{p}(0, \tau), \quad t \in(0, \tau),
$$

satisfying the system

$$
\begin{cases}u^{\prime}(t)=A u(t)+h * B u(t)+f(u(t))+G(t), & t \in(0, \tau), \\ u(0)=u_{0}, & t \in(0, \tau) . \\ \Phi(u)=g(t), & \end{cases}
$$

We assume that (H1), (H2) hold together with the following conditions:

(P1) $B \in \mathcal{L}(D(A), X)$.

(P2) $u_{0} \in D(A)$.

(P3) $\Phi \in X^{\prime}$ and $D\left(A^{\prime}\right)$ is dense in $X^{\prime}$.

(P4) $f \in C^{1}(Y, X)$ and $f^{\prime}: Y \rightarrow \mathcal{L}(Y, X)$ is Lipschitz continuous on the bounded subsets of $Y$.

(P5) $G \in W^{1, p}(0, T ; X)$.

(P6) $v_{0}:=A u_{0}+f\left(u_{0}\right)+G(0) \in(X, D(A))_{1-1 / p, p}$.

(P7) $\Phi\left(B u_{0}\right) \neq 0$.

(P8) $g \in W^{2, p}(0, T)$ with $\Phi\left(u_{0}\right)=g(0)$ and $\Phi\left(v_{0}\right)=g^{\prime}(0)$.

(P9) $f^{\prime}: Y \rightarrow \mathcal{L}(Y, X)$ is bounded, with $f^{\prime}$ Fréchet derivative of $f$.

The following theorems are proved in [29].

Theorem 6. (Local in time existence). Let the assumptions (H1), (H2) and (P1)-(P8) hold. Then there exists $\tau \in(0, T]$, depending on the data, such that the inverse problem given by Definition 4 has a solution $(u, h) \in\left[W^{2, p}(0, \tau ; X) \cap W^{1, p}(0, \tau ; D(A))\right] \times L^{p}(0, \tau)$. 
Theorem 7. (Global in time uniqueness). Let the assumptions (H1), (H2) and (P1)-(P8) hold. Then, if $\tau \in(0, T]$, and the inverse problem given by Definition 4 has two solutions $\left(u_{j}, h_{j}\right) \in\left[W^{2, p}(0, \tau ; X) \cap W^{1, p}(0, \tau ; D(A))\right] \times L^{p}(0, \tau) \quad(j \in\{1,2\})$, then $u_{1}=u_{2}$ and $h_{1}=h_{2}$.

Theorem 8. (Global in time existence and uniqueness). Let the assumptions (H1), (H2) and $(\mathrm{P} 1)-(\mathrm{P} 9)$ hold. Let $T>0$. Then the inverse problem given by Definition 4 has a unique solution $(u, h) \in\left[W^{2, p}(0, T ; X) \cap W^{1, p}(0, T ; D(A))\right] \times L^{p}(0, T)$.

\subsection{A Model in the Theory of Combustion}

Definition 5. Let $T>0$. Determine $\tau \in(0, T]$ and

$$
u \in W^{2, p}(0, \tau ; X) \cap W^{1, p}(0, \tau ; D(A)), \quad k \in L^{p}(0, \tau),
$$

satisfying the system

$$
\left\{\begin{array}{lr}
u^{\prime}(t)=A u(t)+h * B u(t)+f(u(t))+\Phi_{0}\left[u^{\prime}(t)\right] f_{0}, & t \in(0, \tau), \\
u(0)=u_{0}, & t \in(0, \tau) . \\
\Phi(u(t))=g(t), &
\end{array}\right.
$$

We solve the inverse problem under the following conditions on the data. Let $p \in(1,+\infty)$ and let $X, Y, D(A)$ Banach spaces such that (H1) and (H2) hold and assume that:

(Q1) $B \in \mathcal{L}(D(A), X)$.

(Q2) $u_{0} \in D(A), \quad f_{0} \in X$.

(Q3) $\Phi_{0}, \Phi \in X^{\prime}$ and $D\left(A^{\prime}\right)$ is dense in $X^{\prime}$.

(Q4) $f \in C^{1}(Y, X)$ and $f^{\prime}: Y \rightarrow \mathcal{L}(Y, X)$ is Lipschitz continuous on the bounded subsets of $Y$.

(Q5) $\Phi_{0}\left[f_{0}\right] \neq 1$. We set $\chi:=\left(1-\Phi_{0}\left[f_{0}\right]\right)^{-1}$.

(Q6) $\Phi\left[B u_{0}\right]+\chi \Phi\left[f_{0}\right] \Phi_{0}\left[B u_{0}\right] \neq 0$.

(Q7) $v_{0}:=A u_{0}+f\left(u_{0}\right)+\chi \Phi_{0}\left[A u_{0}+f\left(u_{0}\right)\right] f_{0} \in(X, D(A))_{1-1 / p, p}$.

(Q8) $g \in W^{2, p}(0, T)$ with $\Phi\left(u_{0}\right)=g(0)$ and $\Phi\left(v_{0}\right)=g^{\prime}(0)$.

The following theorems are proved in [14].

Theorem 9. (Local in time existence). Let the assumptions (H1), (H2) and (Q1)-(Q8) hold. Then there exists $\tau \in(0, T]$, depending on the data, such that the inverse problem given by Definition 5 has a solution $(u, h) \in\left[W^{2, p}(0, \tau ; X) \cap W^{1, p}(0, \tau ; D(A))\right] \times L^{p}(0, \tau)$.

Theorem 10. (Global in time uniqueness). Let the assumptions (H1), (H2) and (Q1)-(Q8) hold. Then, if $\tau \in(0, T]$, and the inverse problem given by Definition 5 has two solutions $\left(u_{j}, h_{j}\right) \in\left[W^{2, p}(0, \tau ; X) \cap W^{1, p}(0, \tau ; D(A))\right] \times L^{p}(0, \tau) \quad(j \in\{1,2\})$, then $u_{1}=u_{2}$ and $h_{1}=h_{2}$.

Remark 5. In the case the nonlinear function $f(u)$ is sublinear it is possible to prove a global in time existence and uniqueness result for system (37), which is not applicable to the case $f(u)=e^{u}$. The proof follows the ideas developed in [29]. 


\section{Some Applications of the Abstract Results}

\subsection{The Case of Weighted Additional Measurements}

Definition 6. Let $T>0$. Determine $\tau \in(0, T]$ and

$$
u: W^{3, p}\left(0, \tau ; L^{p}(\Omega)\right) \cap W^{2, p}\left(0, \tau ; W^{2, p}(\Omega)\right), \quad h \in L^{p}(0, \tau),
$$

satisfying the system

$$
\left\{\begin{array}{l}
u_{t t}(t, x)=\Delta u_{t}(t, x)+\Delta u(t, x)+h * \Delta u(t, x)+F(u(t, x), \nabla u(t, x))+G(t, x), \\
u(0, x)=u_{0}(x), \quad x \in \Omega, \\
u_{t}(0, x)=u_{1}(x), \quad x \in \Omega, \\
D_{\nu} u(t, x)=0, \quad t \in(0, \tau), x \in \partial \Omega, \\
\int_{\Omega} \phi(x) u(t, x) d x=g(t) .
\end{array}\right.
$$

We solve the inverse problem under the following conditions on the data:

(K1) $\Omega$ is an open bounded subset of $\mathbb{R}^{n}$, lying on one side of its boundary $\partial \Omega$, which is a submanifold of $\mathbb{R}^{n}$ of class $C^{2}$ (in the physical case $n=1,2,3$ ).

(K2) $p \in(1,+\infty), n \in \mathbb{N}$, with $n<p, p \neq 3$.

(K3) $u_{0}, u_{1} \in W_{B}^{2, p}(\Omega):=\left\{u \in W^{2, p}(\Omega): D_{\nu} u \equiv 0\right\}$.

(K4) $\phi \in L^{p^{\prime}}(\Omega)$.

(K5) $f \in C^{1}(\mathbb{R})$ and $f^{\prime}$ is Lipschitz continuous in bounded subsets of $\mathbb{R}$.

(K6) $G \in W^{1, p}\left(0, T ; L^{p}(\Omega)\right)$.

(K7) $v_{1}:=\Delta u_{1}+\Delta u_{0}+f\left(u_{0}, \nabla u_{0}\right)+G(0, \cdot) \in B_{p, p, B}^{2(1-1 / p)}(\Omega)$, where

$$
B_{p, p, B}^{2(1-1 / p)}(\Omega)= \begin{cases}B_{p, p}^{2(1-1 / p)}(\Omega) & \text { if } p<3 \\ \left\{v \in B_{p, p}^{2(1-1 / p)}(\Omega): D_{\nu} v \equiv 0\right\} & \text { if } p>3\end{cases}
$$

(K8) $\int_{\Omega} \phi \Delta u_{0} d x \neq 0$.

(K9) $g \in W^{3, p}(0, T)$ with $\int_{\Omega} \phi u_{0} d x=g(0)$ and $\int_{\Omega} \phi v_{1} d x=g^{\prime}(0)$.

(K10) $f^{\prime}$ is globally bounded.

The following results follow from Theorems 3-5.

Theorem 11. (Local in time existence) Let the assumptions (K1)-(K9) hold. Then there exists $\tau \in(0, T]$, depending on the data, such that the inverse problem given by Definition 6 has a solution $(u, h) \in\left[W^{3, p}\left(0, \tau ; L^{p}(\Omega)\right) \cap W^{2, p}\left(0, \tau ; W^{2, p}(\Omega)\right)\right] \times L^{p}(0, \tau)$.

Theorem 12. (Global in time uniqueness) Let the assumptions (K1)-(K9) hold. Then, if $\tau \in(0, T]$, and the inverse problem given by Definition 6 has two solutions $\left(u_{j}, h_{j}\right) \in$ $\left[W^{3, p}\left(0, \tau ; L^{p}(\Omega)\right) \cap W^{2, p}\left(0, \tau ; W^{2, p}(\Omega)\right)\right] \times L^{p}(0, \tau)(j \in\{1,2\})$, then $u_{1}=u_{2}$ and $h_{1}=h_{2}$.

Theorem 13. (Global in time existence and uniqueness). Let the assumptions (K1)-(H10) hold. Let $T>0$. Then the inverse problem given by Definition 6 has a unique solution $(u, h) \in\left[W^{3, p}\left(0, T ; L^{p}(\Omega)\right) \cap W^{2, p}\left(0, T ; W^{2, p}(\Omega)\right)\right] \times L^{p}(0, T)$. 
4.1.1. The case of flux additional measurements

Definition 7. Let $T>0$. Determine $\tau \in(0, T]$ and

$$
u: W^{3, p}\left(0, \tau ; L^{p}(\Omega)\right) \cap W^{2, p}\left(0, \tau ; W^{2, p}(\Omega)\right), \quad h \in L^{p}(0, \tau),
$$

satisfying the system

$$
\left\{\begin{array}{l}
u_{t t}(t, x)=\Delta u_{t}(t, x)+\Delta u(t, x)+\int_{0}^{t} h(t-s) \Delta u(s, x) d s+\phi(u(t, x), \nabla u(t, x))+\Gamma(t, x), \\
u(0, x)=u_{0}(x), \quad x \in \Omega, \\
u_{t}(0, x)=u_{1}(x), \quad x \in \Omega, \\
D_{\nu} u(t, x)+b(x) u(t, x)=0, \quad(t, x) \in[0, T] \times \partial \Omega, \\
\int_{S}\left(D_{\nu} u_{t}(t, x)+D_{\nu} u(t, x)+h * D_{\nu} u(t, x)\right) \sigma(d x)=g(t) .
\end{array}\right.
$$

For convenience, we define

$$
W_{p}(\Omega):=\left\{u \in W^{2, p}(\Omega): D_{\nu} u(x)+b(x) u(x)=0, \quad x \in \partial \Omega\right\} .
$$

We solve the inverse problem under the following conditions on the data:

(K1) $\Omega$ is an open bounded subset of $\mathbb{R}^{n}$, lying on one side of its boundary $\partial \Omega$, which is a submanifold of class $C^{2}$ of $\mathbb{R}^{n}, b \in C^{1}(\partial \Omega)$.

(K2) $\phi \in C^{1}\left(\mathbb{R}^{n+1}\right)$ and its first order derivatives are Lipschitz continuous in bounded subsets of $\mathbb{R}^{n+1}$.

(K3) $p \in \mathbb{R}, p>n, p \neq 3$.

(K4) $u_{0}, u_{1} \in W_{p}(\Omega)$.

(K5) $\Gamma \in W^{1, p}\left(0, T ; L^{p}(\Omega)\right)$.

(K6) $v_{0}:=\Delta_{x} u_{1}+\Delta_{x} u_{0}+\phi\left(u_{0}, \nabla_{x} u_{0}\right)+\Gamma(0) \in$

$$
\in\left\{\begin{array}{l}
B_{p, p}^{2(1-1 / p)}(\Omega) \text { if } p<3 \\
\left\{v \in B_{p, p}^{2(1-1 / p)}(\Omega): D_{\nu} v+b v=0 \text { in } \partial \Omega\right\} \\
\text { if } p>3 .
\end{array}\right.
$$

(K7) $\int_{S} D_{\nu} u_{0}(x) \sigma(d x) \neq 0$.

(K8) $g \in W^{1, p}(0, T), \quad \int_{S} D_{\nu}\left(u_{0}+u_{1}\right)(x) \sigma(d x)=g(0)$.

(K9) $\nabla \phi$ is bounded in $\mathbb{R}^{n+1}$.

An application of the abstract theorems in [17] gives the following result.

\section{Theorem 14.}

(I) Assume that (K1)-(K8) are satisfied. Then there exists $\tau \in(0, T]$, such that problem given by Definition 7 has a solution $(u, h)$, with

$$
u \in W^{3, p}\left(0, \tau ; L^{p}(\Omega)\right) \cap W^{2, p}\left(0, \tau ; W_{p}(\Omega)\right), \quad h \in L^{p}(0, \tau) .
$$


(II) Assume that (K1)-(K8) are satisfied. If, for some $\tau \in(0, T],\left(u_{1}, h_{1}\right)$ and $\left(u_{2}, h_{2}\right)$ are solutions of problem given by Definition 7, with

$$
\left(u_{1}, h_{1}\right),\left(u_{2}, h_{2}\right) \in\left[W^{3, p}\left(0, \tau ; L^{p}(\Omega)\right) \cap W^{2, p}\left(0, \tau ; W_{p}(\Omega)\right)\right] \times L^{p}(0, \tau),
$$

then $u_{1}=u_{2}, h_{1}=h_{2}$.

(III) Assume that (K1)-(K9) are satisfied. Then problem given by Definition 7 has a unique global solution

$$
(u, h) \in\left[W^{3, p}\left(0, T ; L^{p}(\Omega)\right) \cap W^{2, p}\left(0, T ; W_{p}(\Omega)\right)\right] \times L^{p}(0, T) .
$$

\subsection{The Inverse Problem for the Heat Equation}

Definition 8. Let $T>0$. Determine $\tau \in(0, T]$ and

$$
u: W^{2, p}\left(0, \tau ; L^{p}(\Omega)\right) \cap W^{1, p}\left(0, \tau ; W^{2, p}(\Omega)\right), \quad h \in L^{p}(0, \tau),
$$

satisfying the system

$$
\left\{\begin{array}{l}
u_{t}(t, x)=\Delta u(t, x)+h * \Delta u(t, x)+f(u(t, x))+G(t, x), \\
u(0, x)=u_{0}(x), x \in \Omega \\
D_{\nu} u(t, x)=0, \quad t \in(0, \tau), x \in \partial \Omega \\
\int_{\Omega} \phi(x) u(t, x) d x=g(t)
\end{array}\right.
$$

We solve the inverse problem under the following conditions on the data:

(h1) $\Omega$ is an open bounded subset of $\mathbb{R}^{n}$, lying on one side of its boundary $\partial \Omega$, which is a submanifold of $\mathbb{R}^{n}$ of class $C^{2}$.

(h2) $p \in(1,+\infty), n \in \mathbb{N}$, with $n<p, p \neq 3$.

(h3) $u_{0} \in W_{B}^{2, p}(\Omega):=\left\{u \in W^{2, p}(\Omega): D_{\nu} u \equiv 0\right\}$.

(h4) $\phi \in L^{p^{\prime}}(\Omega)$.

(h5) $f \in C^{1}(\mathbb{R})$ and $f^{\prime}$ is Lipschitz continuous in bounded subsetes of $\mathbb{R}$.

(h6) $v_{0}:=\Delta u_{0}+f\left(u_{0}\right)+G(0) \in B_{p, p, B}^{2(1-1 / p)}(\Omega)$, where

$$
B_{p, p, B}^{2(1-1 / p)}(\Omega)= \begin{cases}B_{p, p}^{2(1-1 / p)}(\Omega) & \text { if } p<3 \\ \left\{v \in B_{p, p}^{2(1-1 / p)}(\Omega): D_{\nu} v \equiv 0\right\} & \text { if } p>3\end{cases}
$$

(h7) $g \in W^{2, p}(0, T)$ with $\Phi\left(u_{0}\right)=g(0)$ and $\Phi\left(v_{0}\right)=g^{\prime}(0)$.

(h8) $\int_{\Omega} \phi(x) \Delta u_{0}(x) d x \neq 0$.

(h9) $G \in W^{1, p}\left(0, T ; L^{p}(\Omega)\right)$.

(h10) $f^{\prime}$ is globally bounded. 
The following results are particular cases of the more general Theorems 18-20 stated just in the sequel.

Theorem 15. (Local in time existence). Let the assumptions (h1)-(h9) hold. Then there exists $\tau \in(0, T]$, depending on the data, such that the inverse problem given by Definition 8 has a solution $(u, h) \in W^{2, p}\left(0, \tau ; L^{p}(\Omega)\right) \cap W^{1, p}\left(0, \tau ; W^{2, p}(\Omega)\right) \times L^{p}(0, \tau)$.

Theorem 16. (Global in time uniqueness). Let the assumptions (h1)-(h9) hold. Then, if $\tau \in(0, T]$, and the inverse problem given by Definition 8 has two solutions $\left(u_{j}, h_{j}\right) \in$ $W^{2, p}\left(0, \tau ; L^{p}(\Omega)\right) \cap W^{1, p}\left(0, \tau ; W^{2, p}(\Omega)\right) \times L^{p}(0, \tau)(j \in\{1,2\})$, then $u_{1}=u_{2}$ and $h_{1}=h_{2}$.

Theorem 17. (Global in time existence and uniqueness). Let the assumptions (h1)-(h10) hold. Let $T>0$. Then the inverse problem given by Definition 8 has a unique solution $(u, h) \in W^{2, p}\left(0, T ; L^{p}(\Omega)\right) \cap W^{1, p}\left(0, T ; W^{2, p}(\Omega)\right) \times L^{p}(0, T)$.

Our main results are proved in an abstract setting so that they can be applied to the more general case of operators of order $2 m$ that contains as a particular case the heat conduction problem for $m=1$.

4.2.1. The inverse problem for operators of order $2 m$

Definition 9. Let $T>0$. Determine $\tau \in(0, T]$ and

$$
u \in W^{2, p}\left(0, \tau ; L^{p}(\Omega)\right) \cap W^{1, p}\left(0, \tau ; W^{2 m, p}(\Omega)\right), \quad h \in L^{p}(0, \tau),
$$

satisfying the system

$$
\left\{\begin{array}{l}
\partial_{t} u(t, x)=A\left(x, \partial_{x}\right) u(t, x)+h * B\left(x, \partial_{x}\right) u(t, x)+\mathcal{F}\left(\left(\partial_{x}^{\alpha} u(t, x)\right)_{|\alpha| \leq 2 m-1}\right)+G(t, x), \\
u(0, x)=u_{0}(x), x \in \Omega, \\
B_{j}\left(x, \partial_{x}\right) u(t, x)=0, \quad 1 \leq j \leq m, t \in(0, \tau), x \in \partial \Omega, \\
\int_{\Omega} \phi(x) u(t, x) d x=g(t) .
\end{array}\right.
$$

We solve the inverse problem under the following conditions on the data:

(k1) $m, n \in \mathbb{N}, \Omega$ is an open bounded subset of $\mathbb{R}^{n}$, lying on one side of its boundary $\partial \Omega$, which is a submanifold of $\mathbb{R}^{n}$ of class $C^{2 m}$.

(k2) $A\left(x, \partial_{x}\right)$ is a strongly elliptic operator of order $2 m$, with coefficients in $C(\bar{\Omega})$, for $j=1, \ldots, m, B_{j}\left(x, \partial_{x}\right)$ is a linear differential operator of order $m_{j} \leq 2 m-1$, with coefficients in $C^{2 m-m_{j}}(\partial \Omega),\left\{B_{j}\left(x, \partial_{x}\right): 1 \leq j \leq m\right\}$ is a normal system of boundary operators, the operator $A\left(x, \partial_{x}\right)$ with vanishing boundary conditions $B_{j}\left(x, \partial_{x}\right)(1 \leq$ $j \leq m)$ has $\operatorname{Arg}(\lambda)=\theta$ as a ray of minimal growth of the resolvent for all $\theta \in$ $[-\pi / 2, \pi / 2]$.

(k3) $p \in(1,+\infty)$, with $n<p, 2 m(1-1 / p) \neq m_{j}+1 / p \quad \forall j=1, \ldots, m$.

$(\mathrm{k} 4) u_{0} \in W_{B}^{2 m, p}(\Omega):=\left\{u \in W^{2 m, p}(\Omega): B_{j}\left(x, \partial_{x}\right) u \equiv 0 \quad \forall j=1, \ldots m\right\}$.

(k5) $\phi \in L^{p^{\prime}}(\Omega)$. 
(k6) $\mathcal{F} \in C^{1}\left(\mathbb{R}^{N(m)}\right)$, with $N(m)$ indicating the cardinality of $\left\{\alpha \in \mathbb{N}_{0}^{n}:|\alpha| ? \leq 2 m-1\right\}$, and we denote by $\left(y_{\alpha}\right)_{|\alpha| ? \leq 2 m-1}$ a general element of $\mathbb{R}^{N(m)}$; moreover, its first order derivatives are Lipschitz continuous on the bounded subsets of $\mathbb{R}^{N(m)}$.

(k7) $G \in W^{1, p}\left(0, T ; L^{p}(\Omega)\right)$.

$(\mathrm{k} 8) v_{0}:=A\left(x, \partial_{x}\right) u_{0}+\mathcal{F}\left(\left(\partial_{x}^{\alpha} u_{0}\right)_{|\alpha| ? \leq 2 m-1}\right)+G(0) \in B_{p, p, B}^{2 m(1-1 / p)}(\Omega)$, where $B_{p, p, B}^{2 m(1-1 / p)}(\Omega):=\left\{v \in B_{p, p}^{2 m(1-1 / p)}(\Omega): B_{j}\left(x, \partial_{x}\right) u \equiv 0, \quad \forall j=1, \ldots m, \quad m_{j}+\right.$ $1 / p<2 m(1-1 / p)\}$.

(k9) $g \in W^{2, p}(0, T)$ with $\Phi\left(u_{0}\right)=g(0)$ and $\Phi\left(v_{0}\right)=g^{\prime}(0)$.

(k10) $B\left(x, \partial_{x}\right)$ is a linear differential operator of order not exceeding $2 m$, with coefficients in $C(\bar{\Omega})$.

(k11) $\int_{\Omega} \phi(x) B\left(x, \partial_{x}\right) u_{0}(x) d x \neq 0$.

(k12) $\nabla \mathcal{F}$ is bounded in $\mathbb{R}^{N(m)}$.

The following results follows from Theorems 6-8.

Theorem 18. (Local in time existence). Let the assumptions $(\mathrm{k} 1)-(\mathrm{k} 11)$ hold. Then there exists $\tau \in(0, T]$, depending on the data, such that the inverse problem given by Definition 9 has a solution $(u, h) \in\left[W^{2, p}\left(0, \tau ; L^{p}(\Omega)\right) \cap W^{1, p}\left(0, \tau ; W^{2 m, p}(\Omega)\right)\right] \times L^{p}(0, \tau)$.

Theorem 19. (Global in time uniqueness). Let the assumptions $(\mathrm{k} 1)-(\mathrm{k} 11)$ hold. Then, if $\tau \in(0, T]$, and the inverse problem given by Definition 9 has two solutions $\left(u_{j}, h_{j}\right) \in$ $\left[W^{2, p}\left(0, \tau ; L^{p}(\Omega)\right) \cap W^{1, p}\left(0, \tau ; W^{2 m, p}(\Omega)\right)\right] \times L^{p}(0, \tau)(j \in\{1,2\})$, then $u_{1}=u_{2}$ and $h_{1}=h_{2}$.

Theorem 20. (Global in time existence and uniqueness). Let the assumptions $(\mathrm{k} 1)-(\mathrm{k} 12)$ hold. Let $T>0$. Then the inverse problem given by Definition 9 has a unique solution $(u, h) \in\left[W^{2, p}\left(0, T ; L^{p}(\Omega)\right) \cap W^{1, p}\left(0, T ; W^{2 m, p}(\Omega)\right)\right] \times L^{p}(0, T)$.

\section{References}

1. Colombo F., Lorenzi A. Identification of Time and Space Dependent Relaxation Kernels in the Theory of Materials with Memory I. Math. Anal. Appl., 1997, no. 213, pp. 32-62.

2. Colombo F., Lorenzi A. Identification of Time and Space Dependent Relaxation Kernels in the Theory of Materials with Memory II. J. Math. Anal. Appl., 1997, no. 213, pp. 63-90. DOI: $10.1006 /$ jmaa.1997.5365

3. Colombo F., Lorenzi A. An Identification Problem Related to a Parabolic Integrodifferential Equation with Noncommuting Spatial Operators. J. Inverse Ill-Posed Probl., 2000, no. 8, pp. $505-540$.

4. Colombo F., Guidetti D., Lorenzi A. Integrodifferential Identification Problems for the Heat Equation in Cylindrical Domains. Adv. Math. Sci. Appl., 2003, no. 13, pp. 639-662.

5. Colombo F., Guidetti D., Lorenzi A. Integrodifferential Identification Problems for Thermal Materials with Memory in Non-Smooth Plane Domains. Dyn. Syst. Appl., 2003, no. 12, pp. $533-560$.

6. Lorenzi A. Direct and Inverse Integrodifferential Maxwell Problems for Dispersive Media Related to Cylindrical Domains. Philadelphia, SIAM, 1995. 
7. Di Cristo M., Guidetti D., Lorenzi A. Abstract Parabolic Equations with Applications to Problems in Cylindrical Space Domains. Adv. Differential Equations, 2010, no. 15, pp. 1-42.

8. Colombo F. Direct and Inverse Problems for a Phase-Field Model with Memory. J. Math. Anal. Appl., 2001, no. 260, pp. 517-545. DOI: 10.1006/jmaa.2001.7475

9. Lorenzi A., Rocca E. Identification of Two Memory Kernels in a Fully Hyperbolic Phase-Field System. J. Inverse Ill-Posed Probl., 2008, no. 16, pp. 147-174. DOI: 10.1515/jiip.2008.010

10. Lorenzi A. An Identification Problem for a Conserved Phase-Field Model with Memory. Math. Methods Appl. Sci., 2005, no. 28, pp. 1315-1339. DOI: 10.1002/mma.614

11. Guidetti D., Lorenzi A. A Mixed Type Identification Problem Related to a Phase-field Model with Memory. Osaka J. Math., 2007, no. 44, pp. 579-613.

12. Colombo F., Guidetti D., Lorenzi A. Identification of Two Memory Kernels and the Time Dependence of the Heat Source for a Parabolic Conserved Phase-Field Model. Math. Meth. Appl. Sci., 2006, no. 28, pp. 2085-2115.

13. Colombo F., Lorenzi A. An Inverse Problem in the Theory of Combustion of Materials with Memory. Adv. Differential Equations, 1998, no. 3, pp. 133-154.

14. Colombo F. An Inverse Problem for a Parabolic Integrodifferential Model in the Theory of Combustion. Physica D, 2007, no. 236, pp. 81-89. DOI: 10.1016/j.physd.2007.07.012

15. Colombo F., Guidetti D. A Unified Approach to Nonlinear Integrodifferential Inverse Problems of Parabolic Type. Z. Anal. Anwendungen, 2002, no. 21, pp. 431-464.

16. Colombo F. An Inverse Problem for the Strongly Damped Wave Equation with Memory. Nonlinearity, 2007, no. 20, pp. 659-683. DOI: 10.1088/0951-7715/20/3/006

17. Colombo F., Guidetti D. Identification of the Memory Kernel in the Strongly Damped Wave Equation by a Flux Condition. Commun. Pure Appl. Anal., 2009, no. 8, pp. 601-620.

18. Bakushinsky A.B., Kokurin M.Yu. Iterative Methods for Approximate Solution of Inverse Problems. Dordrecht, Springer, 2004.

19. Bertero M., Boccacci P. Introduction to Inverse Problems in Imaging. Bristol, Institute of Physics Publishing, 1998. DOI: 10.1887/0750304359

20. Groetsch C.W. Inverse Problems. Activities for Undergraduates. Washington, Mathematical Association of America, 1999.

21. Gladwell G.M.L. Inverse Problems in Vibration. Dordrecht, Kluwer Academic Publishers, 2004.

22. Kabanikhin S.I., Lorenzi A. Identification Problems of Wave Phenomena, Theory and Numerics. Utrecht, VSP, 1999.

23. Isakov V. Inverse Problems for Partial Differential Equations. New York, Springer-Verlag, 1998. DOI: $10.1007 / 978-1-4899-0030-2$

24. Kirsch A. An Introduction to the Mathematical Theory of Inverse Problems. New York, Springer-Verlag, 1996. DOI: 10.1007/978-1-4612-5338-9

25. Kaipio J., Somersalo E. Statistical and Computational Inverse Problems. New York, SpringerVerlag, 2005.

26. Prilepko A.I., Orlovsky D.G., Vasin I.A. Methods for Solving Inverse Problems in Mathematical Physics. New York, Marcel Dekker, Inc., 2000.

27. Romanov V.G. Investigation Methods for Inverse Problems. Utrecht, VSP, 2002. DOI: $10.1515 / 9783110943849$ 
28. Colombo F., Guidetti D. An Inverse Problem for a Phase-Field Model in Sobolev Spaces. Progress in Nonlinear Differential Equations and Their Applications, 2005, no. 64, pp. 189-210.

29. Colombo F., Guidetti D. A Global in Time Existence and Uniqueness Result for a Semilinear Integrodifferential Parabolic Inverse Problem in Sobolev Spaces. Math. Models Methods Appl. Sci., 2007, no. 17, pp. 1-29. DOI: 10.1142/s0218202507002017

30. Lunardi A. Analytic Semigroups and Optimal Regularity in Parabolic Problems. Basel, Birkhauser Verlag, 1995.

31. Triebel H. Interpolation Theory, Function Spaces, Differential Operators. Berlin, Deutscher Verlag der Wissenschaften, 1978.

32. Triebel H. Theory of Functions Spaces. Basel, Birkhauser Verlag, 1983. DOI: 10.1007/978-30346-0416-1

33. Adams R. Sobolev Spaces. New York, Academic Press, 1975.

34. Dore G. Maximal Regularity in $L^{p}$ Spaces for an Abstract Cauchy Problem. Adv. Diff. Eq., 2000, no. 5, pp. 293-322.

35. Hillen T. Qualitative Analysis of Semilinear Cattaneo Equations. Math. Models Methods Appl. Sci., 1998, no. 8, pp. 507-519. DOI: 10.1142/S0218202598000238

Received May 8, 2015

УДК 517.958

DOI: $10.14529 / \mathrm{mmp} 150306$

\title{
О НЕКОТОРЫХ МЕТОДАХ РЕШЕНИЯ ИНТЕГРО-ДИФФЕРЕНЦИАЛЬНЫХ ОБРАТНЫХ ЗАДАЧ ПАРАБОЛИЧЕСКОГО ТИПА
}

\section{Ф. Коломбо}

\begin{abstract}
В статье приводится обзор некоторьх методов решения класса интегродифференциальных обратных задач. А именно, представлены методы решения интегро-дифференциальных обратных задач параболического типа, основанные на теории аналитических полугрупп, результатах по оптимальной регулярности и теореме о неподвижной точке. Эти методы могут быть применены к большому классу физических моделей, например, модели фазового поля, моделей горения, модели сильно затухающих волн с памятью.

Ключевые слова: интегро-дифференциальные обратные задачи; аналитические полугруппы.
\end{abstract}

Фабрицио Коломбо, профессор, кафедра математики, Миланский политехнический университет (г. Милан, Италия), fabrizio.colombo@polimi.it.

Поступила в редакцию 8 мая 2015 2. 